Interview à propos de la SwissFamilyDocs Conference 2013, 29-30 août 2013, BERNEXPO, Berne

\title{
Clairvoyance dans le système complexe de la médecine de famille
}

Interview: Winfried Suske

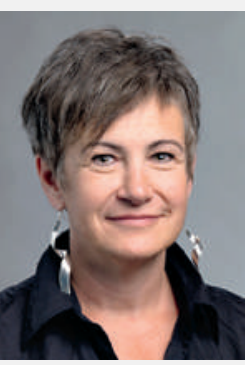

Dr Mireille Schaufelberger (MS)

Directrice de l'institut et de l'enseignement, Institut bernois de médecine de premier recours BIHAM et présidente du Comité de congrès de la SwissFamilyDocs Conference 2013

La formation continue en mutation, nouveau titre de spécialiste en médecine interne générale... Comment la SwissFamilyDocs Conference, avec sa nouvelle approche, satisfait-elle aux nouvelles exigences envers la formation continue dans le domaine des soins de base / de la médecine de famille?

Mireille Schaufelberger (MS): Les limites entre «Médecine interne» et «Médecine interne générale» ne s'estompent pas seulement depuis l'introduction du nouveau titre de spécialiste en médecine interne générale; la médecine interne a toujours été la discipline centrale de la médecine de famille. La SwissFamilyDocs Conference offre aux participants une possibilité de se perfectionner non seulement en ce qui concerne des questions spécifiques issues de la médecine interne générale, mais aussi des questions touchant d'autres domaines médicaux, et ce à un haut niveau et de manière très compacte, soit en l'espace de deux jours récompensés par de nombreux crédits. Le congrès propose en outre un large éventail de connaissances spécifiques axées sur la pratique médicale - allant de l'apprentissage et du perfectionnement d'aptitudes pratiques pour des tâches médicales élargies, les conséquences du nouveau «skill mix» sur la pratique, en passant par de nouveaux modèles de soins et d'organisation de cabinet jusqu'à la compétence croissante dans le domaine de la gestion. En ce faisant, la SFD Conference met les collègues travaillant dans les soins médicaux de base en position de mieux réagir aux nouveaux défis dans le système de santé.

r Winfried Suske

Healthworld (Schweiz) AG

Sennweidstrasse 46

CH-6312 Steinhausen

winfried.suske[at]healthworld.ch
François-Gérard Héritier (FGH): En fait, cette large palette de formation continue s'inscrit dans un continuum cohérent avec la formation pré- puis postgrade qui identifie les rôles du médecin non seu-

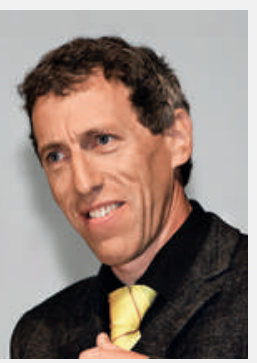

Dr François-Gérard Héritier (FGH)

Président de la Société Suisse de Médecine Générale SSMG et président du Comité de congrès de la SwissFamilyDocs Conference 2013 lement comme expert dans sa spécialité, mais aussi comme un professionnel de la communication, un manager collaborant, un promoteur et un enseignant de la santé.

La SFD Conference est donc une manifestation de formation continue et dans le domaine interniste et dans celui de la médecine de famille? En tant que telle, qu'est-ce qui la rend attractive?

FGH: Lors de la SFD Conference, toute la diversité des tableaux cliniques que nous rencontrons dans le cabinet de médecine de famille et qui rend notre profession tellement passionnante sera évoquée. Outre les sujets internistes, il s'agit également de problèmes chirurgicaux, orthopédiques, gynécologiques et pédiatriques. Les nombreux conférenciers de renom, dont des spécialistes travaillant en cabinet ou en hôpital provenant de la Suisse et d'ailleurs, promettent un programme scientifique de haute qualité, qui sera commenté et complété du point de vue de la médecine de famille.

La combinaison décrite auparavant de sujets touchant la médecine interne et la médecine de famille ainsi que les conférences souvent présentées par des équipes de deux reflètent également la coopération interdisciplinaire tellement importante dans la médecine de famille. L'interdisciplinarité vécue lors de la SFD Conference rend attractif non seulement le congrès, mais surtout la profession de médecin de famille.

Qui sont les personnes qui profitent d'une participation à la SwissFamilyDocs et pourquoi?

MS: La SFD Conference s'adresse en premier lieu à tous les médecins de famille ainsi qu'à toute per- 
sonne souhaitant le devenir. Ces derniers sont d'une part les médecins-assistants avec comme objectif de formation la médecine de premier recours, mais d'autre part aussi les médecins-assistants n'ayant pas encore déterminé leur futur champ d'activité et hésitant entre différentes spécialités. De plus, les chefs de clinique et les étudiants bénéficient également d'une participation, la SwissFamilyDocs Conference leur permettant de se faire une image complète et animée de la médecine de famille!

FGH: Aussi les chefs de clinique - internistes ou représentants d'une discipline spécialisée - pensant s'établir prochainement en cabinet, peuvent apprendre quelque chose à la SwissFamilyDocs Conference. Pourquoi? Parce que le congrès est axé sur la pratique, également en ce qui concerne le côté administratif ou entrepreneurial de la profession de médecin. De plus, les rencontres avec les praticiens en cabinet médical peuvent apporter des réponses concrètes aux questions d'installation. A cet égard, les étudiants sont bien évidemment aussi cordialement invités à visiter la SFD Conference.

\section{Le congrès est axé sur la pratique, également en ce qui concerne le côté administratif ou entrepreneurial de la profession de médecin.}

Lorsqu'il est question de la pensée interdisciplinaire dans l'environnement hospitalier ou au sein des autres disciplines spécialisées, accorde-t-on à la médecine de famille l'importance qu'elle mérite? Qu'est-ce que la SFD Conference apporte à ce sujet?

FGH: Un congrès pour des médecins de famille avec la participation active de toutes sortes de spécialistes est une excellente opportunité de collaboration et d'amélioration de l'interdisciplinarité.

MS: Un nombre de plus en plus élevé d'interfaces est observable dans le domaine des soins médicaux de base - non seulement entre les secteurs ambulatoire et hospitalier, mais aussi entre la médecine de premier recours et les autres disciplines spécialisées. Il est donc souhaitable que nous nous échangions davantage avec nos interlocuteurs en cabinet ou à l'hôpital, et ce au-delà de l'organisation optimale des soins interdisciplinaires. La SwissFamilyDocs Conference donne à tout le monde un bon aperçu du champ de travail et des exigences de la médecine de famille. Ainsi, le programme du congrès fournit également des arguments pertinents pour intégrer, à l'avenir, la médecine de famille dans l'enseignement de tous les futurs médecins.

\section{触, SWISSFAMILYDOCS \\ C O N F E R E N C E 20013}

Qu'est-ce qui rend particulièrement attrayant le programme du congrès 2013?

MS: Sous la direction des JHaS, nous proposons pour la première fois un programme spécialement conçu pour médecins-assistants ainsi que pour étudiants intéressés à la médecine de famille (le jeudi, resp. vendredi après-midi). Egalement pour la première fois, les cinq instituts universitaires de médecine de premier recours contribueront tous à la réussite du congrès avec leurs propres manifestations. Ainsi, la recherche de/dans le cabinet de médecine de famille et l'enseignement dans la médecine de premier recours - mot-clé Teachers Teaching - trouveront également leur place dans le programme.

FGH: Un autre plus consiste, comme mentionné auparavant, dans la grande variété médicale des manifestations proposées. Pour la première fois, le Skill Lab «Motivational Interviewing» donnera la possibilité de s'exercer à un entretien avec un patient acteur. Les Skill Labs sur l'ultrasonographie (pour débutants et experts) ainsi que sur l'exploration fonctionnelle pulmonaire sont également très prometteurs.

Que se cache-t-il derrière la devise du congrès «Retour vers le futur»? Que pouvons-nous apprendre du passé qui nous aide à faire avancer la médecine de premier recours? Quelle est la stratégie avec laquelle assurer l'avenir de la médecine de famille?

MS: Si nous voulons retrouver le chemin vers une médecine de famille forte et nous imposer dans l'avenir, nous devons de nouveau prendre conscience de nos compétences et les défendre avec assurance. Ce retour est facilité par les 30 ans d'existence de la médecine de premier recours universitaire à Berne, que nous fêterons entre autres à l'occasion du congrès 2013. Le pont vers le futur, quant à lui, sera établi par l'implication active, déjà mentionnée, de médecinsassistants et d'étudiants dans l'élaboration du programme ainsi que par l'invitation de la jeune génération au congrès - une contribution à l'encouragement de la relève. En tant que conférenciers, les futurs médecins de famille disposant de premières expériences pratiques se trouvent dans une position solide pour convaincre leurs collègues de l'attractivité de leur profession. Nous tenons également beaucoup à soigner le contact avec la jeune génération de médecin de famille et à lui donner tout l'appui possible - si elle le souhaite. 
FGH: Nous voulons réaffirmer que la médecine de famille a un futur malgré les turbulences qu'elle traverse. Une relève se prépare, elle va travailler différemment et elle peut s'appuyer sur les valeurs des anciens, ouverts à de nouveaux modèles, avec toujours le patient au centre.

Et comment les parties du programme de médecine interne reprennent-elles la devise du congrès?

MS: Plusieurs contenus ou sujets de conférences explorent explicitement des technologies d'avenir ou des technologies déjà réelles, notamment «Le checkup du futur», "Gentests in der Hausarztpraxis», «Innovationsmanagement», ou «Skill Mix - wie viel Teameinsatz braucht es wirklich in der Hausarzt-

\section{L'interdisciplinarité vécue lors de la SFD Conference rend attractif non seulement le congrès, mais surtout la profession de médecin de famille.}

praxis?», «Médecine 2.0: Internet, le médecin et son patient» et «Neue Infektionskrankheiten in der Schweiz durch Klimawandel». Afin de nous prémunir pour l'avenir proche, la SFD Conference propose de nombreux séminaires de rafraîchissement et de mise à jour médicale.

Quelle est l'importance du «skill mix», discuté au congrès, pour les médecins de famille?

FGH: Ce «skill mix» est typiquement un thème d'actualité qui devrait prendre de plus en plus d'importance dans la pratique médicale de nos jeunes générations. Pour relever les défis de l'augmentation des maladies chroniques et de la complexité croissante des prises en charges médicales, combinés aux attentes et aux envies de pratique de nos jeunes collègues surtout féminines, la réponse est certainement un partage des compétences et du travail en partena- riat avec les autres professionnels de santé. Mais tous ces modèles ont besoin d'un pilote, le médecin de famille. Il est donc primordial que nos congrès s'intéressent à la formation du meilleur pilote possible.

MS: Les médecins de premier recours doivent définir de façon proactive leurs compétences et déterminer leur rôle et leurs tâches au sein du système de santé futur à l'aide de ces compétences spécifiques à la médecine de famille. Nous souhaitons encourager et soutenir ce processus à la SFD Conference.

Quelles impulsions espérez-vous recevoir de cette $3^{e}$ SwissFamilyDocs Conference, pour la médecine de famille suisse et pour les futurs congrès SFD?

FGH: Notre formation est et doit rester continue. Chaque congrès apporte une pierre à cette construction. Cette $3^{\text {e }}$ SFD Conference devrait contribuer à l'édification d'une médecine de famille novatrice, ouverte, confiante en ses possibilités et orientée vers le futur.

MS: Il serait souhaitable qu'avec le temps, toutes les organisations impliquées dans la médecine de famille participent activement à la SwissFamilyDocs Conference. Une médecine de premier recours bien interconnectée est forte, et la SFD Conference devrait le refléter de façon convaincante pour tous les partis, y compris la politique.

Personnellement, de quoi vous réjouissez-vous le plus, en vue de ce congrès en août?

FGH: Je me réjouis de la curiosité d'acquérir de nouvelles connaissances et du plaisir de rencontrer des collègues plus jeunes et moins grisonnant(e)s.

MS: Je me réjouis de la présence de nombreux médecins-assistants et étudiants et de l'échange avec nos futurs collègues, afin de donner à notre relève potentielle une idée des côtés extrêmement passionnants de la médecine de famille.

Pour d'ultérieures informations au sujet du congrès et des inscriptions veuillez visiter www.swissfamiliydocs.ch 